\title{
MRI VISUALIZATION OF SMALL STRUCTURES USING IMPROVED SURFACE COILS
}

\author{
Manuel Rivera,* Juan José Vaquero, ${ }^{\dagger}$ Andrés Santos,* \\ Jesús Ruiz-Cabello, $\$$ Francisco del Pozo* \\ *Grupo de Bioingeniería y Telemedicina, Universidad Politécnica de Madrid, Madrid, Spain, †Nuclear Medicine \\ Department, National Institutes of Health, Bethesda, MD USA, and †Unidad RMN. Instituto Pluridisciplinar, \\ Universidad Complutense de Madrid, Madrid, Spain
}

\begin{abstract}
In this paper we present the spatial resolution enhancement and noise reduction level achieved with an optimized inductively coupled surface coil specificall designed for our experiments. The technique of designing and implementing customized coils for magnetic resonance imaging of very small structures is described. We have designed a low cost prototype of an inductively coupled circular surface coil, tuned for ${ }^{1} \mathrm{H}$ magnetic resonance imaging at $200 \mathrm{MHz}$. The coil is mounted on a customized teflo support. The inductive coupling used in this coil improves the signal-to-noise ratio by reducing various loss mechanisms (specially the dielectric losses). Test images have been acquired to determine the evolution of induced articular lesions in a rabbit animal model, as well as brain tumors in rats. The images show high spatial resolution, excellent $B_{1}$ fiel homogeneity and no "hot spots". Comparing these images with those acquired with conventional coils, one find better spatial resolution and signal-to-noise ratio, as well as larger fiel of view with less intense illumination artifact. The methodology can be used in any application that requires high quality imaging of small structures.
\end{abstract}

Keywords: Surface coil; High resolution MRI; Inductively coupled coils; Low noise images.

\section{INTRODUCTION}

Magnetic resonance (MR) imaging of small structures is a valuable tool in studies of laboratory models such as evolution of induced articular lesions in rabbits, or the assessment of brain tumor development in rats. To visualize and quantify these processes, we needed to develop better probes for our imaging equipment. This paper presents our approach to the problem; the results indicate that the proposed solution surpass the performance of-the-shelf coils.

The MR image is created by decoding the radiofrequency signal obtained from the induced voltage in the receiver coil due to the precession of the magnetization vector. ${ }^{1-5}$ This signal (s) is contaminated by undesired noise (r) at the detector input. The relationship between signal and noise is described by a dimensionless variable known as the signal-to-noise ratio (SNR); the higher the SNR is, the better the image quality. ${ }^{6,7}$
Since the objects involved in our studies are very small, quantitative analysis of their properties requires a very high SNR in order to provide sharp images and enough contrast between the region of interest under study and the surrounding structures.

Surface coils (SC) are widely used in MRI, since they improve resolution when visualizing superficia structures, achieving a high SNR over the region adjacent to the coil. SC also enhance the SNR with respect to the body coils over its Field of View (FOV) and, due to its inherent higher spatial selectivity, it is possible to improve the spatial resolution on the visualization of small structures. ${ }^{11,12}$

In the Theory section, we describe the basic physic principles applied to the SC designs. In the Material and Methods section, our SC implementation itself, as well as the settings and subjects for the experiments are described. Images and measurements are presented in the
ReCEIVED 7/3/97; ACCEPTED 10/10/97.

Address correspondence to Juan José Vaquero, Nuclear Medicine Department, National Institutes of Health, Bldg. 10,
Room 1C401, Bethesda, MD 20892-1180. E-mail: juanjo@ nmdhst.cc.nih.gov 


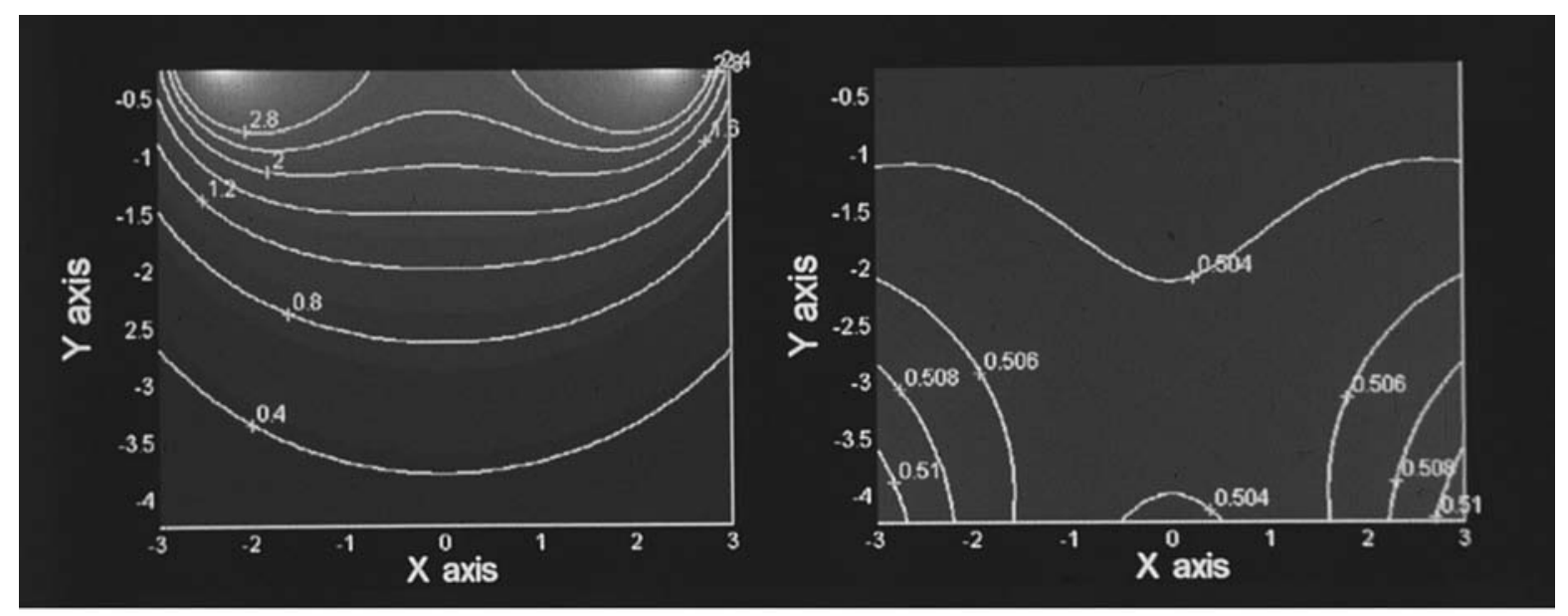

a

b

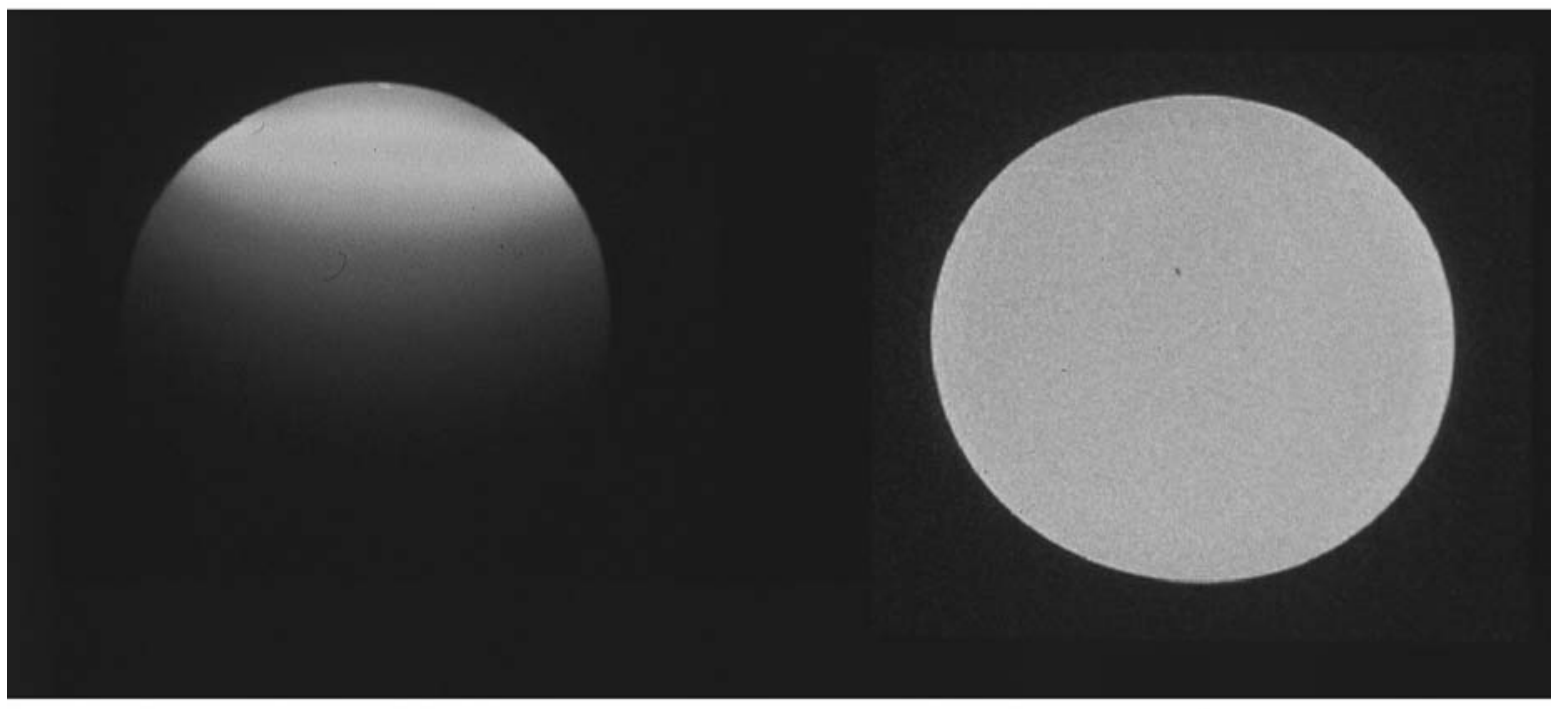

C

d

Fig. 1. (a) Sensitivity map in arbitrary units of a $48 \mathrm{~mm}$ one-turn circular SC lying in $\mathrm{Y}=0$ plane. The simulation has been realized in $Z=0$ plane. It can be shown that the sensitivity is highest at the proximity of the coil. (b) Sensitivity map in $Z=0$ for a body coil ("saddle coil") with a length of $40 \mathrm{~cm}$ and a diameter of $60 \mathrm{~cm}$. (c) Image of a $50 \mathrm{~mm}$ diameter sphere fille with water in a $\mathrm{Z}=0$ plane. It can be remarked the correlation between the sensitivity map and the image. Slice thickness is $1 \mathrm{~mm}$. (d) Image of the sphere with the body coil. Slice thickness was increased to $5 \mathrm{~mm}$ to achieve the same SNR that in image (c).

Results section, and we conclude with a Discussion about those results.

\section{THEORY}

\section{The Signal}

The signal induced in the reception coil by the $\mathrm{i}$-th voxel of a tissue is proportional to:

$$
\mathrm{S}_{\mathrm{i}} \propto\left(\mathrm{M}_{\mathrm{xy}}\right)_{\mathrm{i}} \cdot\left(\mathrm{B}_{1 \mathrm{xy}}\right)_{\mathrm{i}}
$$

where $\left(\mathrm{M}_{\mathrm{xy}}\right)_{\mathrm{i}}$ is the transversal component of the magnetization vector of the voxel and $\left(\mathrm{B}_{1 \mathrm{xy}}\right)_{\mathrm{i}}$ is the sensitiv- ity of the receiver coil at the position where the voxel is placed. According to the Reciprocity Principle, the sensitivity is computed as the transversal component of the magnetic fiel which would be induced by the receiver coil if it were working as a transmitter coil driven by a unit current. ${ }^{1,6}$

SC have the inconvenience of having a different sensitivity at each point depending on the depth. ${ }^{1-3}$ If the correlation between the obtained image and the tissues real distribution has to be high (image fidelity) the sensitivity map must be as uniform as possible. Ideally, the sensitivity should be the same everywhere in such a 


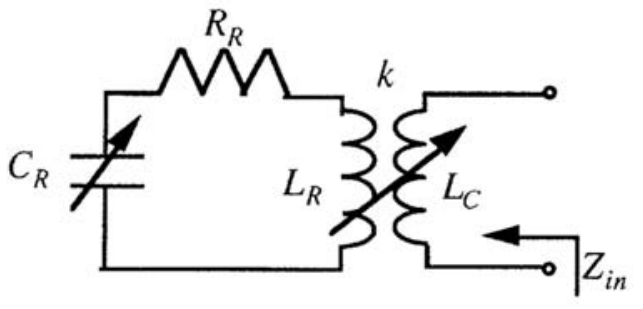

(a)

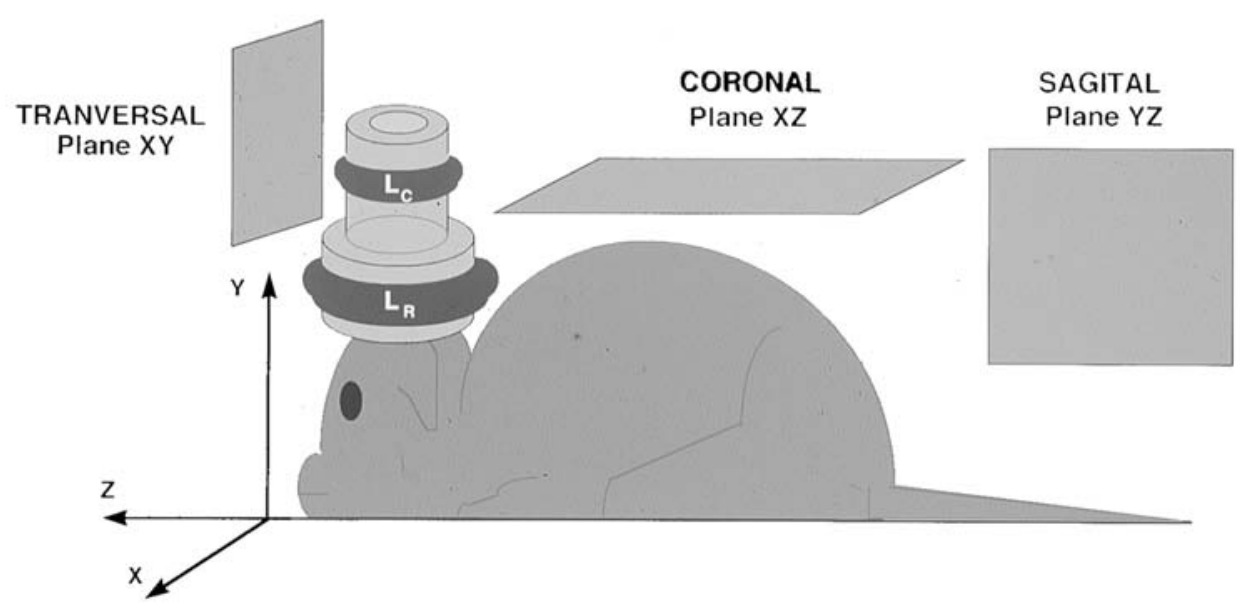

(d)

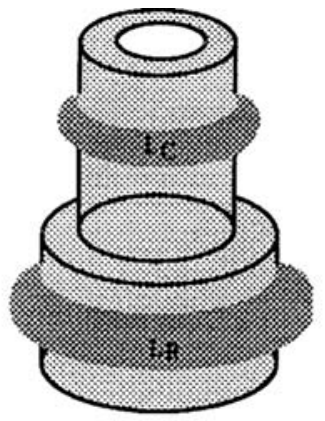

(b)

CORONAL

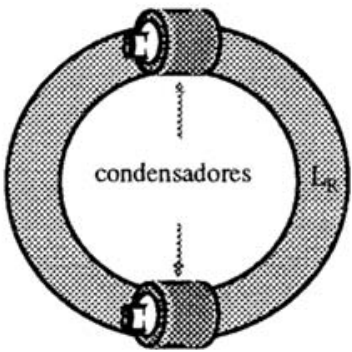

(c)

Fig. 2. (a) Equivalent circuit of an inductively coupled SC. The matching is realized by varying the separation between the loops, while the tuning is done with the capacitor $\mathrm{C}_{R}$. (b) Inductively coupled SC GBT-SC-1. $\mathrm{L}_{R}$ is the receiver loop and $\mathrm{L}_{C}$ the coupling loop. (c) Detail of the $\mathrm{L}_{\mathrm{R}}$ loop with the different tuning capacitors distributed along the coil. (d) Coil placement on the sample when used on rat brain studies.

way that the image would only depend on the sample characteristics. SC sensitivity non-homogeneity, named intensity or illumination artifact, causes a modulation on the image brightness in such a way that the image does not only depend on the sample, but also on the geometry and position of the SC itself (Fig. 1).

Another inconvenience of SC is that the sample must be placed as near as possible to the coil because the sensitivity decreases with distance (Fig. 1a). Body coils do not have this problem since their sensitivity is almost constant over the imaged plane, as depicted in Fig. 1b. However, these coils have a lower sensitivity than the SC, and therefore they lead to a smaller resolution, mainly due to the necessity of increasing the slice thickness in order to get similar signal intensity with the same sample.

Finally, it is convenient to design SC adapted to the region of interest and by means of computer simulations estimate the sensitivity map to place the sample where the homogeneity is highest.

\section{Noise}

Noise voltage is calculated as the square-root of total power dissipated by the different loss mechanisms present in a MR system. We will only consider the two most important sources of noise: coil losses (or resistive losses) and sample losses.

Coil losses are due to the skin effect which increases with the frequency. ${ }^{8}$ These losses diminish when the conductor cross-section used to build the receiver coil is increased, and when conductors with smaller resistivity are used.

Sample losses are caused by two different mechanisms, inductive and dielectric. The inductive losses, which are present in every nuclear magnetic resonance (NMR) experiment, are due to the radiofrequency magnetic fiel power dissipation. This oscillating magnetic fiel heats the sample, and if the dissipated power at any point of it is high, a local increase of the temperature is produced (a so-called "hot spot"), which can lead to the sample damage. ${ }^{9,10}$ 
Table 1. Comparison of the signal-to-noise ratio (SNR) for different images of a water phantom

\begin{tabular}{|c|c|c|c|c|c|c|c|c|c|c|c|}
\hline $\begin{array}{l}\text { Slice } \\
(\mathrm{mm})\end{array}$ & $\begin{array}{c}\text { Pixel } \\
\left(\mathrm{mm}^{3}\right)\end{array}$ & Probe & $\begin{array}{c}\text { Att } \\
(\mathrm{dB})\end{array}$ & $\overline{\mathrm{x}}_{\mathrm{s}}$ & $\sigma_{\mathrm{r}}$ & $\begin{array}{c}(\mathrm{SNR})_{1} \\
\text { (nat) }\end{array}$ & $\begin{array}{c}(\mathrm{SNR})_{1} \\
(\mathrm{~dB})\end{array}$ & A & $\sigma$ & $\begin{array}{c}(\mathrm{SNR})_{2} \\
\text { (nat) }\end{array}$ & $\begin{array}{c}(\mathrm{SNR})_{2} \\
(\mathrm{~dB})\end{array}$ \\
\hline 0.5 & 0.027 & commercial & 0 & 0.912 & 0.035 & 26.07 & 14.16 & 0.910 & 0.053 & 17.05 & 12.31 \\
\hline 0.5 & 0.027 & GBT-SC-1 & 3 & 0.940 & 0.016 & 58.78 & 17.69 & 0.930 & 0.024 & 38.51 & 15.85 \\
\hline 1 & 0.055 & commercial & 2 & 0.984 & 0.019 & 51.84 & 17.15 & 0.916 & 0.027 & 33.93 & 15.30 \\
\hline 1 & 0.055 & GBT-SC-1 & 7 & 0.936 & 0.010 & 93.64 & 19.71 & 0.920 & 0.015 & 61.38 & 17.88 \\
\hline 2 & 0.110 & commercial & 6 & 0.979 & 0.011 & 88.98 & 19.49 & 0.990 & 0.017 & 58.28 & 17.65 \\
\hline 2 & 0.110 & GBT-SC-1 & 12 & 0.975 & 0.008 & 121.93 & 20.86 & 0.959 & 0.012 & 79.98 & 19.02 \\
\hline 3 & 0.165 & commercial & 9 & 0.978 & 0.009 & 108.69 & 20.36 & 0.952 & 0.013 & 73.25 & 18.65 \\
\hline 3 & 0.165 & GBT-SC-1 & 15 & 0.820 & 0.006 & 136.78 & 21.36 & 0.897 & 0.010 & 89.70 & 19.52 \\
\hline
\end{tabular}

Details in text.

pixel vol. Pixel volume.

Att. Attenuation in $\mathrm{dB}$ of the amplifie gain receiver.

$\overline{\mathrm{x}}_{\mathrm{s}}$ Signal mean value.

$\sigma_{\mathrm{r}}$ Noise standard deviation.

A Signal amplitude.

$\sigma$ Noise.

$(\mathrm{SNR})_{1}$ Signal-to-noise ratio calculated as the quotient of the homogeneous signal mean value and the standard deviation of image noise.

$(\mathrm{SNR})_{2}$ Signal-to-noise ratio calculated with the expressions obtained by Henkelman.

(nat) Signal-to-noise ratio in natural units.

(dB) Signal-to-noise ratio in decibels.

The dielectric losses are caused by voltage differences among different parts of the receiver coil that produce an electrostatic fiel around the coil. ${ }^{7,8,11}$ Dielectric losses cause detuning of the receiver circuit, and therefore the reduction of the received signal. These losses can be reduced by designing a balanced SC, which allows improvement of up to $75 \%$ compared to the equivalent, unbalanced design. The results are better still if the tuning capacitance is distributed along the coil, ${ }^{11}$ by placing several equivalent distributed capacitors instead of using only one. Tuning and matching procedures of commonly used SCs are implemented using two or three tunable capacitor components. With such an approach, it is very difficul to build a balanced circuit.

Nowadays, inductively coupled SC are being developed for different applications, solving some of the problems mentioned above. The main advantage of inductively-coupled SCs is their balanced structure that reduces the dielectric losses. ${ }^{11,12}$ In addition, matching is achieved by varying the coupling between coils. For this reason, such designs are also known as single-parameter adjustable probes, since only a tuning capacitor needs to be used (Fig. 2a). Following these guidelines, we have designed and built a series of probes that use inductive coupling architecture. Our surface coils (GBT-SC probes) presented in the following paragraphs generate very high quality images.

\section{MATERIAL AND METHODS}

\section{System and Probes}

All images were acquired using a $4.7 \mathrm{~T}$ Bruker (BIOSPEC BMT-47/40) system, so the Larmor Frequency for protons is $200 \mathrm{MHz}$. Probes work in the single-coil transmit/receive mode. For this system we built an inductively-coupled SC (GBT-SC-1), which consists on two silver-plated copper turns wound on a teflo support (Fig. 2b), providing a good coupling between them. The tubular shape of the support for the coils allows the samples to be placed near to the coil, fillin the area where the homogeneity is highest. The receiver coil $\mathrm{L}_{R}$ is a $48 \mathrm{~mm}$ diameter one-turn built with a $4 \mathrm{~mm}$ silverplated copper wire. The tuning capacitor $\mathrm{C}_{\mathrm{R}}$ has been distributed along the coil to diminish the dielectric losses as we indicated previously. Two tuning capacitors have been used: a non-magnetic chip and a variable capacitor (Fig. 2c). The coupling loop $\mathrm{L}_{\mathrm{C}}$ was built with a $30 \mathrm{~mm}$ diameter one-turn silver-plated loop with a $2 \mathrm{~mm}$ copper section. Another design was developed to acquire images of rabbit knees. This probe (GBT-SC-2) consists of a 30 $\mathrm{mm}$ diameter $\mathrm{L}_{\mathrm{R}}$ and was designed to fi the shape of the sample; it has the same structure as the GBT-SC-1.

We will compare the quality of the images acquired with a general-purpose probe provided by the manufacturer and our GBT-SC probes. The commercial probe is a $48 \mathrm{~mm}$ diameter one-turn built with a $4 \mathrm{~mm}$ silverplated copper wire (same diameter than GBT-SC-1). The electronic circuit is a non-balanced structure, with variable capacitors for tuning and matching. For animal studies, a stereotaxic support was built in our laboratory to position rat heads immediately below the coil plane.

\section{Subjects and Images}

For image quality measurements, SNR is computed for three different objects: a phantom, a Wistar rat and a 
Table 2. Comparison of the signal-to-noise ratio (SNR) for different images of a wistar rat head

\begin{tabular}{|c|c|c|c|c|c|c|c|c|c|c|c|c|}
\hline Sect & $\begin{array}{l}\text { Slice } \\
(\mathrm{mm})\end{array}$ & $\begin{array}{l}\text { Pixel } \\
\left(\mathrm{mm}^{3}\right)\end{array}$ & Probe & $\begin{array}{l}\text { Att } \\
(\mathrm{dB})\end{array}$ & $\overline{\mathrm{x}}_{\mathrm{s}}$ & $\sigma_{\mathrm{r}}$ & $\begin{array}{l}(\mathrm{SNR})_{1} \\
\text { (nat) }\end{array}$ & $\begin{array}{c}(\mathrm{SNR})_{1} \\
(\mathrm{~dB})\end{array}$ & A & $\sigma$ & $\begin{array}{c}(\mathrm{SNR})_{2} \\
\text { (nat) }\end{array}$ & $\begin{array}{c}(\mathrm{SNR})_{2} \\
(\mathrm{~dB})\end{array}$ \\
\hline $\mathrm{c}$ & 1 & 0.038 & commercial & -3 & 0.426 & 0.043 & 9.99 & 9.99 & 0.422 & 0.065 & 6.48 & 8.12 \\
\hline $\mathrm{c}$ & 1 & 0.038 & GBT-SC-1 & 3 & 0.402 & 0.017 & 23.52 & 13.71 & 0.403 & 0.026 & 15.45 & 11.89 \\
\hline $\mathrm{c}$ & 2 & 0.072 & commercial & 2 & 0.510 & 0.024 & 21.02 & 13.22 & 0.512 & 0.037 & 13.81 & 11.40 \\
\hline $\mathrm{c}$ & 2 & 0.072 & GBT-SC-1 & 9 & 0.451 & 0.012 & 37.44 & 15.73 & 0.453 & 0.018 & 24.61 & 13.91 \\
\hline $\mathrm{c}$ & 3 & 0.114 & commercial & 5 & 0.465 & 0.018 & 25.30 & 14.03 & 0.466 & 0.028 & 16.61 & 12.20 \\
\hline $\mathrm{c}$ & 3 & 0.114 & GBT-SC-1 & 12 & 0.504 & 0.010 & 49.48 & 16.94 & 0.506 & 0.016 & 32.54 & 15.12 \\
\hline $\mathrm{t}$ & 1 & 0.038 & commercial & -3 & 0.447 & 0.037 & 12.13 & 10.84 & 0.446 & 0.562 & 7.92 & 8.99 \\
\hline $\mathrm{t}$ & 1 & 0.038 & GBT-SC-1 & 3 & 0.355 & 0.015 & 23.03 & 13.62 & 0.355 & 0.024 & 15.11 & 11.79 \\
\hline $\mathrm{t}$ & 2 & 0.072 & commercial & 2 & 0.429 & 0.019 & 22.71 & 13.56 & 0.429 & 0.029 & 14.9 & 11.73 \\
\hline $\mathrm{t}$ & 2 & 0.072 & GBT-SC-1 & 9 & 0.350 & 0.009 & 41.33 & 16.16 & 0.351 & 0.013 & 27.19 & 14.34 \\
\hline $\mathrm{t}$ & 3 & 0.114 & commercial & 5 & 0.358 & 0.013 & 26.91 & 14.30 & 0.359 & 0.020 & 17.67 & 12.47 \\
\hline $\mathrm{t}$ & 3 & 0.114 & GBT-SC-1 & 12 & 0.509 & 0.007 & 74.28 & 18.70 & 0.511 & 0.011 & 48.82 & 16.88 \\
\hline
\end{tabular}

Details in text.

sect. Section: coronal (c), transversal (t).

pixel vol. Pixel volume.

Att. Attenuation in $\mathrm{dB}$ of the amplifie gain receiver.

$\overline{\mathrm{x}}_{\mathrm{s}}$ Signal mean value.

$\sigma_{\mathrm{r}}$ Noise standard deviation.

A Signal amplitude.

$\sigma$ Noise.

(SNR) 1 Signal-to-noise ratio calculated as the quotient of the homogeneous signal mean value and the standard deviation of image noise.

$(\mathrm{SNR})_{2}$ Signal-to-noise ratio calculated with the expressions obtained by Henkelman.

(nat) Signal-to-noise ratio in natural units.

(dB) Signal-to-noise ratio in decibels.

rabbit. For the phantom, Fast Spin-Echo images were acquired using the Rapid Acquisition with Relaxation Enhancement (RARE) sequence. ${ }^{13}$ Five consecutive slices with different thickness were obtained $(0.5,1,2$ and $3 \mathrm{~mm}$ ) for both probes, using the FOV of $60 \mathrm{~mm}$ and a $256 \times 256$ acquisition matrix. Coronal and transverse images of a rat head were also acquired with both probes, using the RARE sequence. The FOV was $50 \mathrm{~mm}$ and a $256 \times 256$ acquisition matrix was used. Slice thickness was $1 \mathrm{~mm}$, repetition time (TR) $=1500 \mathrm{~ms}$, echo time $(\mathrm{TE})=20 \mathrm{~ms}$, and 1 number of excitations (NEX). Three consecutive oblique slices, at a $23^{\circ}$ angle from a coronal slice, were acquired with the MultiSlice-MultiEcho-MSME-sequence, using the GBT-SC-1 probe. The FOV was $60 \mathrm{~mm}$ and the acquisition matrix was $256 \times$ 256; the slice thickness was $1 \mathrm{~mm}, \mathrm{TR}=500 \mathrm{~ms}, \mathrm{TE}=$ $25 \mathrm{~ms}$, and 1 NEX. The images of a rabbit knee were acquired with the GBT-SC-2 coil, using a RARE sequence with a FOV of $25 \mathrm{~mm}$, and the acquisition matrix of $256 \times 256$, slice thickness of $0.5 \mathrm{~mm}, \mathrm{TR}=2000 \mathrm{~ms}$, $\mathrm{TE}=13 \mathrm{~ms}$, and NEX $=8$. Both animals (Wistar rat and rabbit) were anaesthetized i.p. with Ketolar $(10 \mathrm{mg} / \mathrm{Kg})$.

\section{Measurements}

Both signal and noise were measured over a square region of interest $(\mathrm{ROI}), 20 \times 20$ pixels size. For the evaluation of the signal, the ROI was selected in the region of highest homogeneity, to minimize the impact of the SCs sensitivity profil on the mentioned data. For the noise estimation, the ROI was placed in a corner of the FOV, on the image background. This minimized the effect of the sensitivity profil of the SC since in that location there is no magnetic fiel induced (Fig. 1a).

SNR was computed in two different ways:

$\mathrm{SNR}_{1}$. $-\mathrm{A}$ ratio of the mean signal value $\overline{\mathrm{x}}_{\mathrm{S}}$ and the standard deviation of noise $\sigma_{\mathrm{r}}$ was calculated:

$$
\mathrm{SNR}_{1}=\frac{\overline{\mathrm{x}}_{\mathrm{s}}}{\sigma_{\mathrm{r}}}
$$

$\mathrm{SNR}_{2}$. - Computed according to Henkelman. ${ }^{14}$ This computation is valid only for magnitude images reconstructed with the direct Fourier method, and it considers correction factors to obtain the amplitude signal in presence of noise:

$$
\mathrm{SNR}_{2}=\frac{\mathrm{A}}{\sigma} \quad \sigma=\frac{\sigma_{\mathrm{r}}}{0.655} \quad \mathrm{~A}=\sqrt{\overline{\mathrm{x}}_{\mathrm{s}}^{2}+\sigma_{\mathrm{s}}^{2}-2 \cdot \sigma^{2}}
$$

where A is the signal amplitude, $\sigma$ the noise, $\sigma_{\mathrm{r}}$ the noise standard deviation, $\overline{\mathrm{x}}_{\mathrm{s}}$ the signal mean value, and $\sigma_{\mathrm{s}}$ the signal standard deviation.

\section{RESULTS}

Once the coils were built and tuned, the quality factors (Q) were measured at $200 \mathrm{MHz}$ with an RF network 


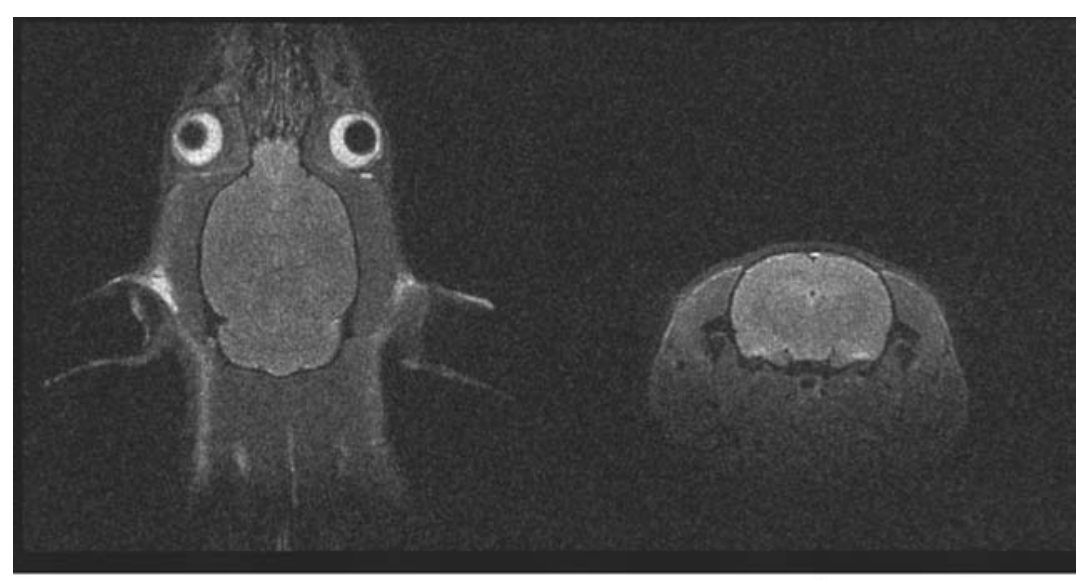

a

b

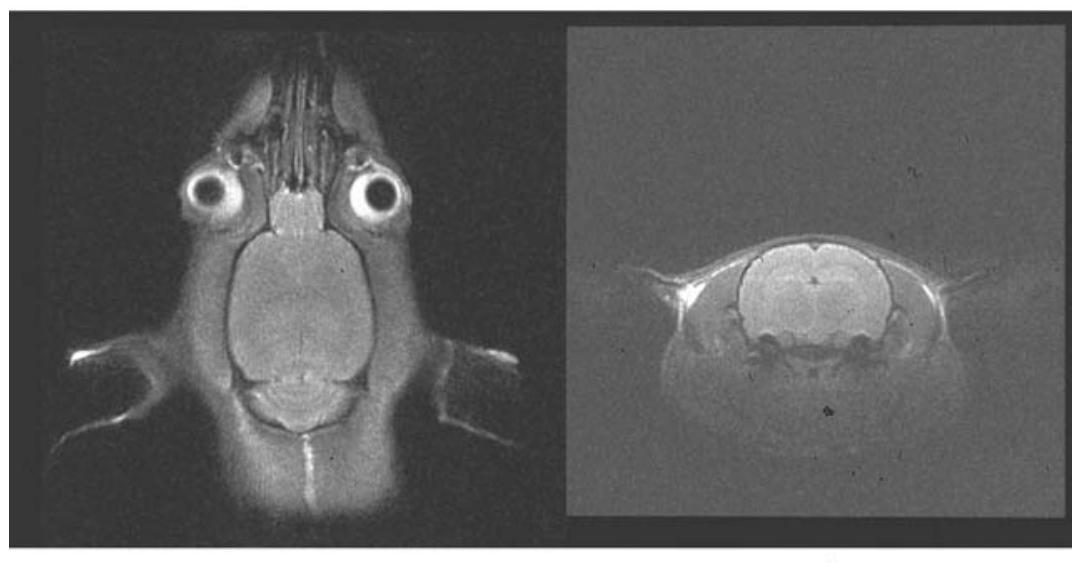

C

d

Fig. 3. Images of a rat head acquired with the RARE sequence: (a) and (b) with the commercial probe, (c) and (d) with the GBT-SC-1 probe. (a) and (c) are coronal slices and (b) and (d) are transversal slices. Images acquired with the commercial probe present a high level of noise. Details in text.

analyzer (Hewlett-Packard model 8711A). For the GBTSC- 1 the unloaded Q is 500, and loaded with the phantom (a $50 \mathrm{~mm}$ diameter sphere fille with water) is 375 . GBT-SC-2 has a $\mathrm{Q}$ factor equal to 600 when unloaded, and 425 when loaded with the same phantom. For the standard commercial probe used in our comparisons, the $\mathrm{Q}$ values are 415 (unloaded) and 250 (loaded).

Figure $2 \mathrm{~d}$ shows the coil placement on the animal for rat brain studies. The special design of the support for our probes allows us to place the sample where the sensitivity map is more intense and homogeneous (Fig. 1c). Because of the spherical shape of the samples (phantom, rat head and rabbit knee) the GBT-SC probes are placed as a crown over them, making it possible to increase the FOV and the homogeneity by exploring the symmetry of the sensitivity map with respect to the plane of the SC. The commercial probe is inside a $2 \mathrm{~mm}$-thick plastic box, so it was placed over the sample.
We have evaluated the performance of our probes and the commercial one by comparing the SNR, homogeneity and spatial resolution. The results obtained with the phantom images are summarized in Table 1. To improve statistical significance data in Table 1 were calculated as the mean value over several consecutive slices. Table 2 summarizes the results of our coil and compares them with the commercial one when imaging rat brains. The SNR of images acquired with GBT-SC coils (Figs. 3-5) is twice that of images acquired with the commercial coil, for a slice thickness of $0.5 \mathrm{~mm}$.

Figure 6 presents the images and the normalized sensitivity profile for a $0.5 \mathrm{~mm}$ slice thickness acquired with both probes. The 128 pixels profile across the marked line have been normalized to remove the effect of the different attenuations set by the system during the acquisition. Our GBT-SC-2 probe was also used to obtain high resolution images of a rabbit knee, being the slice thickness $0.5 \mathrm{~mm}$ (Fig. 7). 


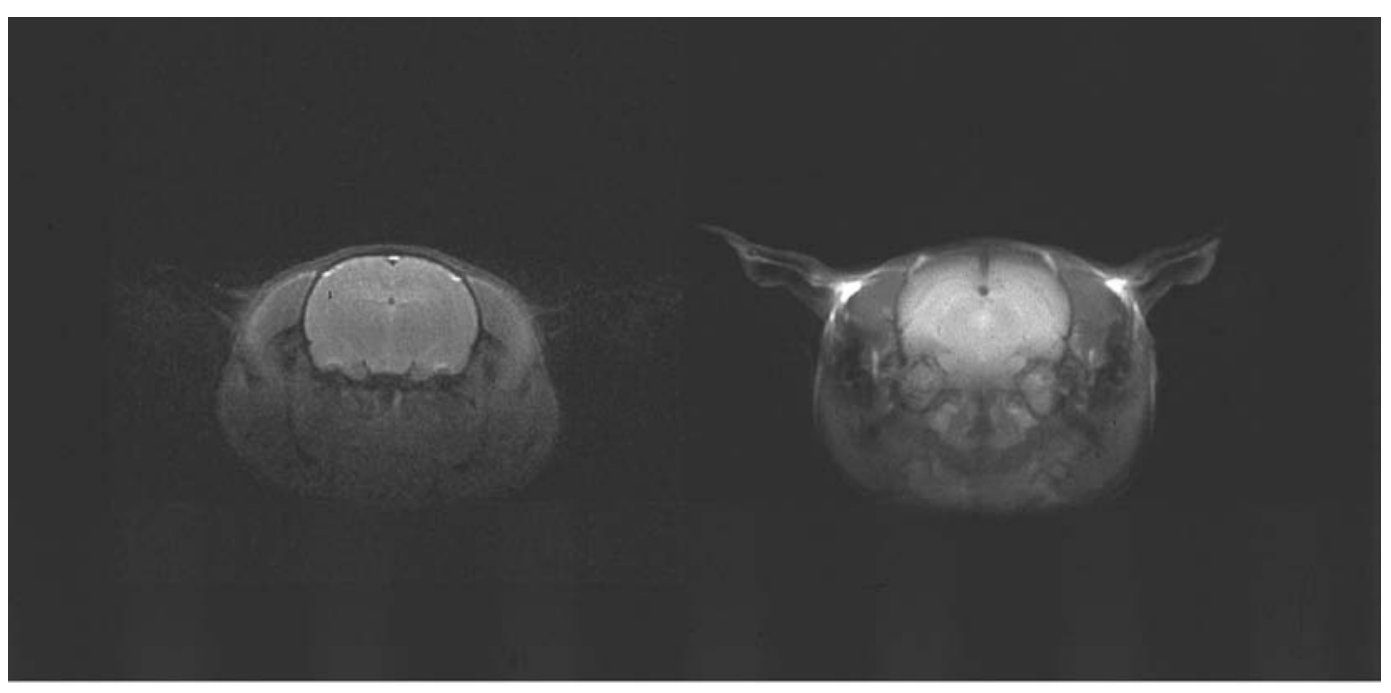

a

b

Fig. 4. Image of a rat head acquired with the RARE sequence: (a) commercial probe, (b) GBT-SC-1 probe. The image (a) presents an important intensity artifact. This effect disappears in image (b), which is very homogeneous. Details in text.

\section{DISCUSSION}

The conclusions derived from the previous tables are summarized here. 1) In all images the SNR is higher when the GBT-SC probes are used. 2) Image improvement obtained with the proposed SC is higher for thinner slices. 3) As can be seen in Fig. 6, the relative noise level has been drastically reduced with the GBT-SC-1 probe; the image (right) also presents less variations in adjacent pixels than the one obtained with the commercial probe. This suggests that the illumination artifact has been reduced. 4) There is an additional indicator of the better quality of the GBT-SC probes over the commercial ones: The MR equipment works with a constant radiofrequency amplificatio gain, and it adjusts a programmable attenuator connected at its input to avoid receiver saturation, allowing an optimized digitization of the signal. When the level of the detected signal increases, a higher attenuation is programmed automatically by the system: the GBT-SC probes have required a higher attenuation than the standard SC, which means that their sensitivity is higher. This indirect measurement together with the fact that SNR has been improved confirm that the signal levels are higher with our probes than with the standard ones, verifying the principles applied on the design. 5) The normalized level of noise is always smaller for our probes. There are two reasons for these results: the balanced circuit design (it is this firs stage the one that mostly influence the noise figure) and the signal atten-

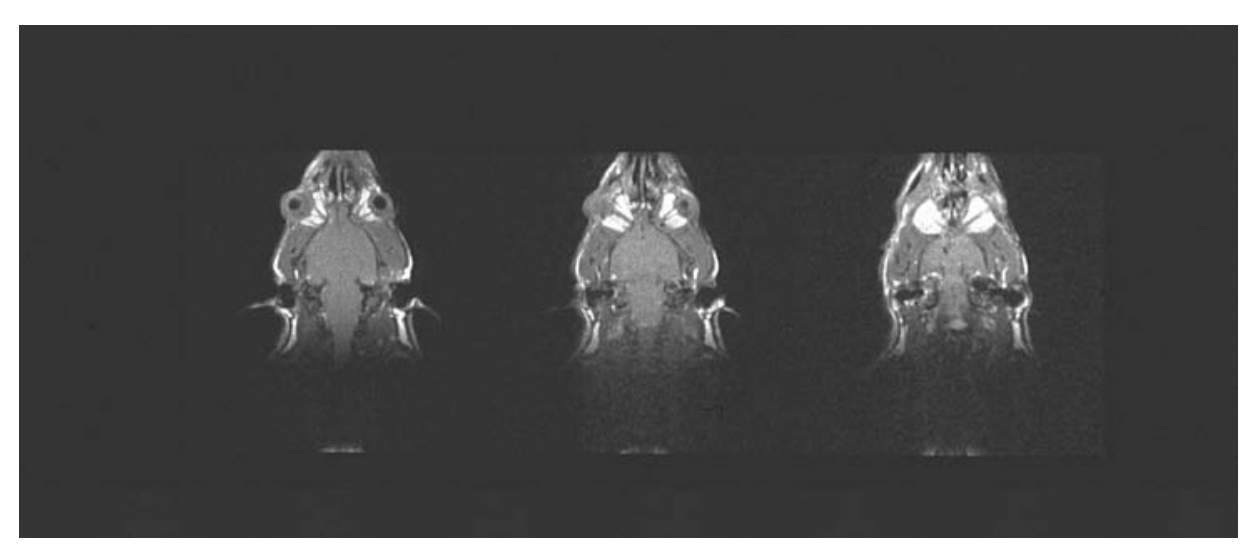

Fig. 5. Images of a rat optical nerve acquired with the MSME sequence and the GBT-SC-1 probe. The slices are consecutive, being deeper the left slice. Details in text. 


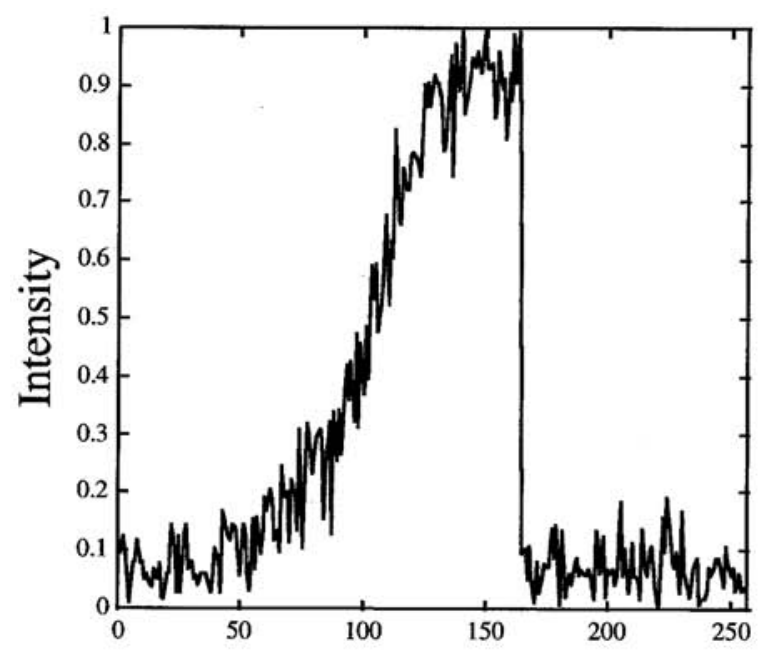

Pixels

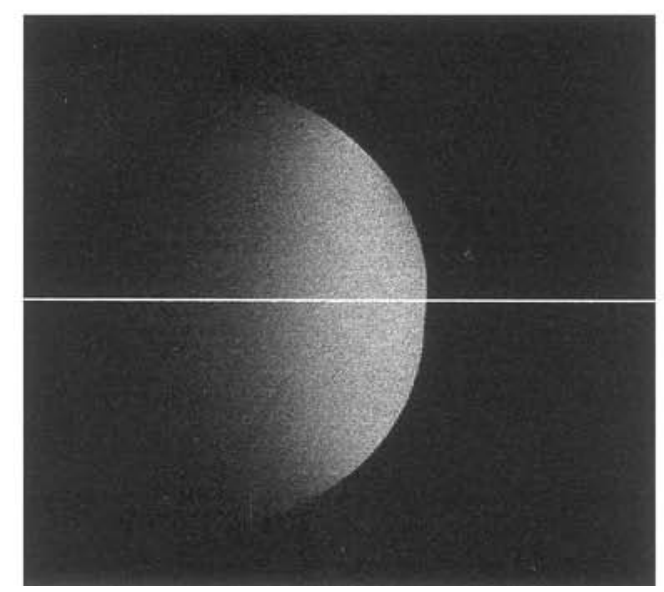

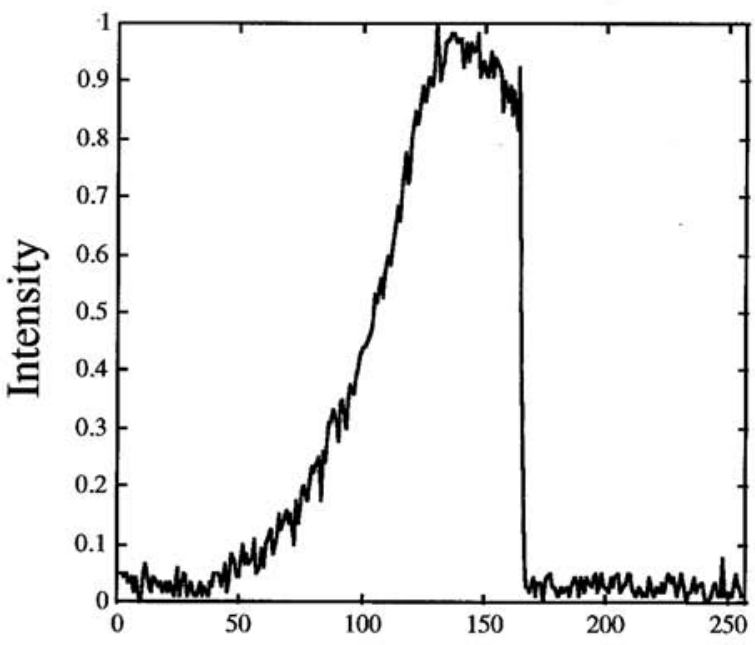

Pixels

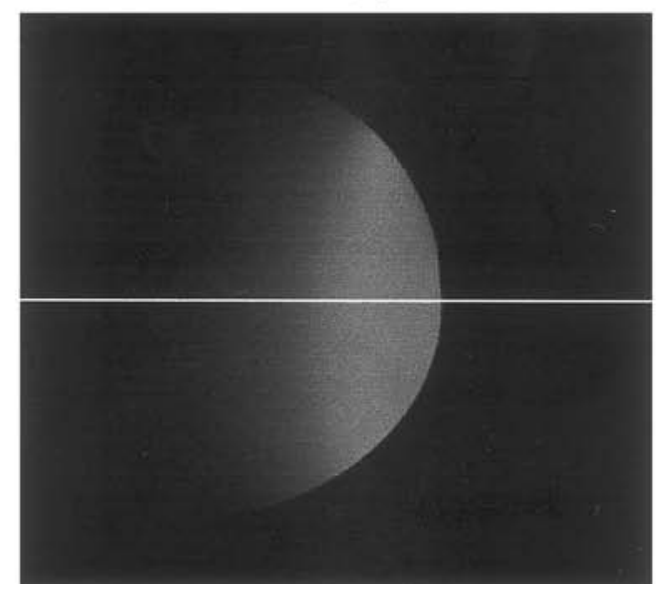

Fig. 6. Normalized intensity profile of a $0.5 \mathrm{~mm}$ thickness slice obtained with the standard probe (left) and with the GBT-SC-1 (right). The sample is a $50 \mathrm{~mm}$ diameter sphere fille with water. Profile show the severe noise level reduction achieved with the proposed probe.

uation at the input amplifier 6) Theoretically, the SNR should vary linearly with slice thickness for the same probe; this does not happen in our results. A cause for this observation can be that for very thin slices, the signal and the noise levels become similar, and then the standard deviation of the noise is relatively high, deteriorating the SNR further than the lineal prediction. However, the attenuation level applied to the input of the firs amplifie increases with the slice thickness, which means that the received signal intensity has increased as expected; this explains the apparent reduction in the received signal intensity.

\section{Homogeneity}

A visual analysis of the images points out a better homogeneity in the images acquired with GBT-SC probes. Intensity artifact has also been reduced (Fig. 3 and Fig. 4). Examples of these improved results are the images obtained with the GBT-SC-1: three consecutive slices of the rat optical nerve, with a thickness of $1 \mathrm{~mm}$ (Fig. 5), where it is possible to distinguish perfectly the optical nerve from its beginning at the blind spot to its end in the connection (optic chiasm) with the encephalon.

\section{High Resolution}

Rabbit knee images depict an excellent quality that allows a perfect observation of the very small structures studied in the induced cartilage degeneration experiment for which the probes were developed. These kinds of images could not be obtained with the commercial probe.

FOV

GBT-SC have an improved FOV. We were able to acquire signals from tissues localized at $22 \mathrm{~mm}$ depth while with the standard probe the maximal depth 


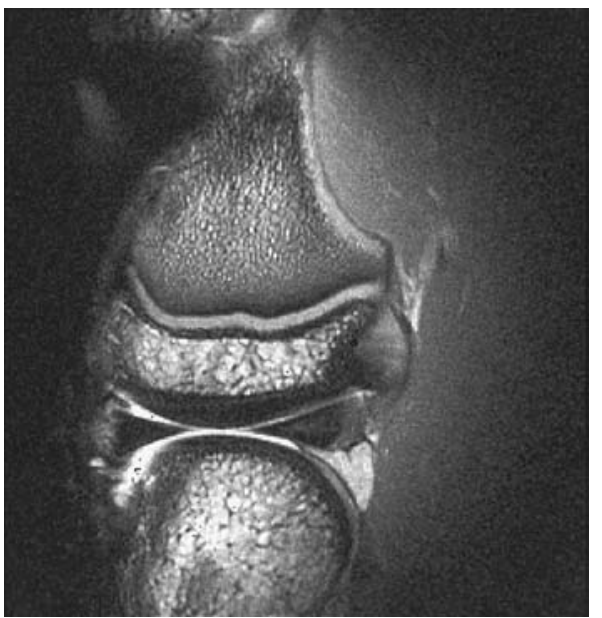

Fig. 7. Image of a rabbit knee acquired with the RARE sequence and the GBT-SC-2 probe. Details in text.

reached was $15 \mathrm{~mm}$ (Fig. 4). Thus, we could observe the entire rat head with a strongly reduced intensity artifact.

\section{CONCLUSION}

Surface coils may solve many of the scientifi and clinical problems that require high resolution or high performance imaging methods. Our aim in this communication was to demonstrate that by combining an adequate geometrical design and an optimization of the coil structure and electronic circuits, we were able to implement a probe that improves image quality for high resolution of small animal imaging. The importance of these results is not relying on a particular improvement in the electronics; in fact, the technique applied is well known and used (inductive coupling). However, our report describes how the optimization of coil performance can lead to a significan improvement in MRI quality.

With the results presented here, we have demonstrated the image quality improvement obtained with the GBT-SC. The general purpose, commercial probe has two major drawbacks when used to image the microstructures we were interested in: 1) The minimum slice thickness required to achieve good SNR of the acquired image is $2 \mathrm{~mm}$. With the commercial probe it was not possible to reduce the voxel volume without degradation of the image resolution (Fig. 3). 2) The FOV depth is less than $15 \mathrm{~mm}$. This implies that all the tissues located deeper could not be observed (Fig. 4). When a probe is not designed for a specifi application, it is not possible to place it as near as possible to the sample, especially where the sensitivity and the homogeneity are higher, reducing the FOV and increasing the intensity artifact respectively.

By adapting the SC geometrical design to the region of study, and by implementing the electronics (tuning and matching circuits) to reduce all losses mechanisms, our GBT-SC-1 and GBT-SC-2 produce MR images that demonstrate their superiority over the general-purpose probe.

With this design we have been able to achieve greater image quality, high resolution MRI of small structures. We have demonstrated that with a clear specificatio of the problem and a detailed knowledge of the region of interest, state-of-the-art technology allows a better result. These conclusions obtained with animal and phantom images are easily extended to a broad range of applications for human studies in clinical MRI systems.

Acknowledgments-The authors thank the members of the NMR Unit at the Instituto Pluridisciplinar (Universidad Complutense de Madrid), in particular Marien Fernández-Valle and Margarita Izquierdo, and Ching-Nien Chen of the National Institutes of Health (Bethesda, MD, USA) for her advice. J.J. Vaquero acknowledges the support of CICYT (Spanish Government). J. Ruiz-Cabello acknowledges the support of CICYT (Spanish Government), grant BIO97-0543.

\section{REFERENCES}

1. Hoult, D.I.; Richards, R.E. The signal-to-noise ratio of the nuclear magnetic resonance experiment. J. Magn. Reson. 71:85-24; 1976.

2. Hoult, D.I.; Lauterbur, P.C. The sensitivity of the zeugmatographic experiment involving human samples. J. Magn. Reson. 425:433-34; 1985.

3. Evelhoch, J.L.; Crowley, M.G.; Ackerman, J.J.H. Signalto-noise optimization and observed volume localization with circular surface coils. J. Magn. Reson. 110:124-56; 1984.

4. Chen, C-N.; Hoult, D.I. Signal and Noise. Biomedical Magnetic Resonance Technology. Bristol and New York: Adam Hilger, 1989: pp. 117-145.

5. Mansfield P.; Morris, P.G. Some Hardware Considerations. NMR Imaging in Biomedicine. New York: Academic Press, 1982: pp. 289-297.

6. Sobol, W.T. Dedicated coils in magnetic resonance imaging. Rev. of Magn. Reson. Med. 181:224-1; 1986.

7. Murphy, M.K.; Fernandez, E.J.; Clark, D.S. A comparison of three radiofrequency coils for NMR studies of conductive samples. Magn. Reson. Med. 382:389-12; 1989.

8. Ramo, S.; Whinnery, J.R.; van Duzer, T. Fields and Waves in Communication Electronics. New York: J. Wiley \& Sons, 1965: pp. 178-183.

9. Budinger, T.F. Health effects of in vivo nuclear magnetic resonance. IEEE Eng. in Med. and Biol. Magazine: 31:38; 1985.

10. Shellock, F.G. MRI Biologic Effects and Safety Consid- 
erations. In: Higgins C.B.; Hricak, H.; Helms, C.A. (Eds). Magnetic Resonance Imaging of the Body. New York: Raven Press 1992: pp. 233-265.

11. Decorps, M.; Blondet, P.; Reutenauer, H.; Albrand, J.P.; Remy, C. An inductively coupled, series-tuned NMR probe. J. Magn. Reson. 100:109-65; 1985.

12. Froncisz, W.; Jesmanowicz, A.; Hyde, J.S. Inductive (flu linkage) coupling to local coils in magnetic resonance imaging and spectroscopy. J. Magn. Reson. 135:143-66; 1986.

13. Henning, J.; Nauhert, A.; Friedburg, H. RARE imaging: a fast imaging method for clinical MR. Magn. Reson. Med. 823:833-3; 1986.

14. Henkelman, M. Measurement of signal intensities in the presence of noise in MR images. Med. Phys. 232:233-12; 1985. 